\title{
Formation of giant planets around stars with various masses
}

\author{
K. Kornet ${ }^{1,2}$, S. Wolf ${ }^{1}$, and M. Różyczka ${ }^{2}$ \\ 1 Max Planck Institute for Astronomy, Königstuhl 17, 69117 Heidelberg, Germany \\ e-mail: kornet@camk.edu.pl \\ 2 Nicolaus Copernicus Astronomical Center, Bartycka 18, Warsaw 00-716, Poland \\ Received 23 June 2005 / Accepted 7 March 2006
}

ABSTRACT

\begin{abstract}
We examine the predictions of the core accretion - gas capture model concerning the efficiency of planet formation around stars with various masses. First, we follow the evolution of gas and solids from the moment when all solids are in the form of small grains to the stage when most of them are in the form of planetesimals. We show that the surface density of the planetesimal swarm tends to be higher around less massive stars. Then, we derive the minimum surface density of the planetesimal swarm required for the formation of a giant planet both in a numerical and in an approximate analytical approach. We combine these results by calculating a set of representative disk models characterized by different masses, sizes, and metallicities, and by estimating their capability of forming giant planets. Our results show that the set of protoplanetary disks capable of giant planet formation is larger for less massive stars. Provided that the distribution of initial disk parameters does not depend too strongly on the mass of the central star, we predict that the percentage of stars with giant planets should increase with decreasing stellar mass. Furthermore, we identify the radial redistribution of solids during the formation of planetesimal swarms as the key element in explaining these effects.
\end{abstract}

Key words. accretion, accretion disks - planetary systems: protoplanetary disks - planetary systems: formation

\section{Introduction}

Radial velocity surveys led to the discovery of over 150 extrasolar planets around main sequence stars. Published descriptions of most of them can be found in the references given by Marcy et al. (2005) and Mayor et al. (2004). Those surveys have been the most successful in the case of $\mathrm{G}$ dwarf stars, because such stars have well-defined spectroscopic features and show only a little photospheric activity. Consequently, most of the known extrasolar planets orbit stars similar to our Sun. Due to the constant progress in the detection techniques, the observational programs recently started to also include stars with lower masses on a larger scale, namely $\mathrm{M}$ dwarfs. Moreover, some of these surveys are now particularly dedicated to lower-mass stars (e.g. Endl et al. 2003; Bonfils et al. 2004). So far, these efforts have led to the discovery of three planets around two M dwarf stars: Gliese 876b,c (Marcy et al. 1998, 2001) and GJ436b (Butler et al. 2004).

From the theoretical point of view, the problem of giant planet formation around $\mathrm{M}$ dwarfs was studied recently by Laughlin et al. (2004). They addressed it within the core accretion - gas capture model (CAGCM) that provides the most widely accepted scenario explaining the formation of giant planets in both the Solar System and extrasolar planetary systems. This model predicts that first a solid planetary core is formed by collisional accumulation of planetesimals. When the core reaches a mass of a few Earth masses, it starts to accrete gas, and an extended hydrostatic envelope is built around it. As the accretion rate of gas is greater than the accretion rate of solids at this time, the envelope eventually becomes more massive than the core. When this happens, a runaway accretion of gas ensues, which is terminated either by tidal interactions of the planet with the protoplanetary disk or by the dissipation of the disk. CAGCM has found supporting evidence in the discovery that stars with planets have higher metallicities than field stars (Santos et al. 2000; Fischer \& Valenti 2003). This is because the formation time of giant planets decreases with increasing surface density of the planetesimal swarm (Pollack et al. 1996), which in turn increases with the primordial metallicity of the protoplanetary disk. Thus, giant planets are expected to form more easily in disks with higher metallicity.

Laughlin et al. (2004) conclude that M dwarfs have a limited ability to form Jupiter-mass planets. This is a direct consequence of their assumption that the surface density of the planetesimal swarm out of which planetary cores are formed scales linearly with the mass of the central star. However, the solid component of the protoplanetary disk evolves in a different way than the gaseous component (Weidenschilling \& Cuzzi 1993). Due to the gas drag, a significant redistribution of solids takes place, and in the inner disk their surface density can be substantially enhanced compared to the initial one (Weidenschilling 2003; Stepinski \& Valageas 1997). In general, the efficiency of the processes responsible for the redistribution of dust depends on the mass of the central star. An obvious conclusion is that analysis of the formation of giant planets around stars with various masses should include the global evolution of solids in protoplanetary disks. A simple model of the evolution of solids was proposed by Kornet et al. (2002); Kornet et al. (2005). Applying it to solarlike central stars, these authors reproduced the observed correlation between stellar metallicity and the probability of a planet occurring (a similar result was independently obtained by Ida $\&$ Lin 2004b).

The rapid progress in observational techniques opens up the possibility of testing the correctness and predictive power of the model proposed by Kornet et al. (2005). To that end, we extend their analysis and calculate probabilities of planet occurrence around stars with different masses (both smaller and larger than 
$1 M_{\odot}$ ), which may be compared to future observational data. In Sect. 2 we explain our approach to the evolution of protoplanetary disks and planet formation. The results of our calculations are presented in Sect. 3 and discussed in Sect. 4.

\section{Methods of calculation}

\subsection{The disk}

We model the protoplanetary disk as a two-component fluid, consisting of gas and solids. The gaseous component is described by the analytical model of Stepinski (1998), which gives the surface density of gas, $\Sigma_{\mathrm{g}}$, as a function of distance $a$ from the star and time $t$, in terms of a selfsimilar solution to the viscous diffusion equation. The viscosity coefficient is given by the standard $\alpha$ prescription:

$v=\frac{1}{3} \alpha C_{\mathrm{S}} H$

where $H$ is a density scale-height of gaseous disk and $C_{\mathrm{S}}$ denotes the speed of sound in the gas. All other quantities characterizing the gas are obtained in a thin disk and vertical thermal balance approximation by solving the set of equations (see, for example Frank et al. 1992):

$\Sigma_{\mathrm{g}}=2 H \rho_{\mathrm{g}}$

$H=\frac{\sqrt{2} C_{\mathrm{s}}}{\Omega_{\mathrm{K}}}$

$C_{\mathrm{s}}^{2}=\frac{k_{\mathrm{B}} T}{\mu m_{\mathrm{H}}}$

$\frac{16 \sigma_{\mathrm{B}} T^{4}}{3 \kappa \Sigma_{\mathrm{g}}}=\frac{9}{4} \Sigma_{\mathrm{g}} \nu \Omega_{\mathrm{K}}^{2}$.

Here $T$ is the temperature in the midplane of the disk, and $\Omega_{\mathrm{k}}$ the Keplerian angular velocity. For the Rosseland mean opacity $\kappa$ the analytical piecewise-continuous power law formulas from Ruden \& Pollack (1991) were adopted. In the parts of the disk where the optical thickness falls below a critical value of $\tau_{\text {crit }}=1.78$ (Ruden \& Pollack 1991), the last equation is replaced by:

$\frac{4 \sigma_{\mathrm{B}} \kappa T^{4}}{3 \tau_{\text {crit }}}=\frac{9}{4} \nu \Omega_{\mathrm{K}}^{2}$.

The main assumptions underlying our approach to the evolution of solids are the folllowing. (1) At each radial distance from the central star the particles have the same size (which in general varies over time). (2) There is only one component of dust, in this case corresponding to high-temperature silicates with the evaporation temperature $T_{\text {evap }}=1350 \mathrm{~K}$ and bulk density $3.3 \mathrm{~g} \mathrm{~cm}^{-3}$. This choice is justified by the fact that in most cases the surface density of a water-ice planetesimal swarm would be too low in the range of distances considered here, e.g. $r \leq 5 \mathrm{AU}$, to enable formation of a giant planet in a reasonable time (see also Kornet et al. 2004). We include other species, e.g. water ice only, in modeling the gaseous disk as the sources of opacity. In other words we assume that the evolution of silicate grains is not strongly influenced by the evolution of i.e. ice grains. (3) All collisions between particles lead to coagulation. (4) When the temperature exceeds $T_{\text {evap }}$, local solids immediately sublimate and the vapour evolves at the same radial velocity as the gas component. (5) Initially, in regions where the disk temperature is lower than $T_{\text {evap }}$, all solids are in the form of grains with radii $10^{-3} \mathrm{~cm}$ (the results do not depend on the choice of that particular value, as long as the solids are initially small enough to be coupled well to the gas). (6) The radial velocities of solid particles are entirely determined by the effects of gas drag. (7) The relative velocities of solid particles when they collide are computed according to the turbulent model described by Stepinski \& Valageas (1997). (8) The evolution of the solids does not affect the evolution of the gas. At each radius, the vertical extent of the solid particle distribution is calculated and is evolved in time, so the effect of the sedimentation of solids toward the midplane of the disk is taken into account. All assumptions and approximations are discussed in Stepinski \& Valageas (1997), Stepinski (1998), and Kornet et al. (2001).

The evolution of solids is governed by two equations. The first of them is the continuity equation for the surface density of solid material, $\Sigma_{\mathrm{s}}$. The second one, describing the evolution of grain sizes, can be interpreted as the continuity equation for sizeweighted surface density of solids, $\Sigma_{\text {size }} \equiv s(a) \Sigma_{\mathrm{s}}$, where $s(a)$ is the radius of solid particles at a distance $a$ from the star. The equations are solved numerically on a moving grid whose outer edge follows the outer edge of the dust disk. The details of the method can be found in Kornet et al. (2001).

\subsection{The planets}

We model the formation of a giant planet in situ, so the orbital parameters of the planet do not vary in time. Our procedure for the evolution of the protoplanetary cores is based on the following assumptions: (1) core accretion starts when solids at a given distance $a$ from the star reach radii of $2 \mathrm{~km}$; (2) at each time, the planetesimals are mixed well through the feeding zone of the planet, so their surface density $\Sigma_{\mathrm{s}}$ is always uniform in space, but usually decreasing with time as planetesimals accrete onto the planet; (3) the planetesimals do not migrate into the feeding zone from outside or inside and vice versa, but they can be overtaken by the boundary of the feeding zone as it expands due to the growing mass of the planet. Under these assumptions the growth of protoplanetary core mass $M_{\mathrm{c}}$ can be described by the formula given by Papaloizou \& Terquem (1999),

$\dot{M}_{\mathrm{c}}=C_{1} C_{\text {cap }} R_{p} R_{H} \Omega_{\mathrm{K}} \Sigma_{\mathrm{s}}$,

where

$R_{\mathrm{H}}=a\left(\frac{M_{\mathrm{p}}}{h M_{\star}}\right)^{1 / 3}$

is the radius of the Hill sphere of the planet. The quantity $h$ is a constant factor that reflects different definitions of the Hill radius in the literature. Herein we assume $h=3$. The value of $C_{1}$ given by Papaloizou \& Terquem (1999) is $81 \pi / 32$; we use a factor of 5 (the difference comes from a different definition of $R_{\mathrm{H}}$ ). The quantity $C_{\text {cap }}$ describes the increase in the effective capture radius of the planet with respect to its real radius $R_{\mathrm{p}}$ due to interaction of planetesimals with the envelope of the planet (Podolak et al. 1988). We approximate it with a fit to the results of Bodenheimer et al. (2000) provided by Hubickyj (2001). For core masses less than $5 M_{\odot}$, no increase in the effective capture radius is assumed, i.e. $C_{\text {cap }}\left(M_{\mathrm{c}}<5 M_{\oplus}\right)=1$. For higher core masses, it increases linearly with the mass of the core, reaching its maximum value of $C_{\text {cap }}=5$ for $M_{\mathrm{c}}=15 M_{\oplus}$. The surface density of planetesimals $\Sigma_{\mathrm{s}}$ also changes in time, as they are accreted onto the core, and the feeding zone expands. Under our assumptions it can be calculated as

$\Sigma_{\mathrm{s}}=\Sigma_{\mathrm{s}, \text { init }}-\frac{M_{\mathrm{c}}}{2 \pi a \Delta a}$ 

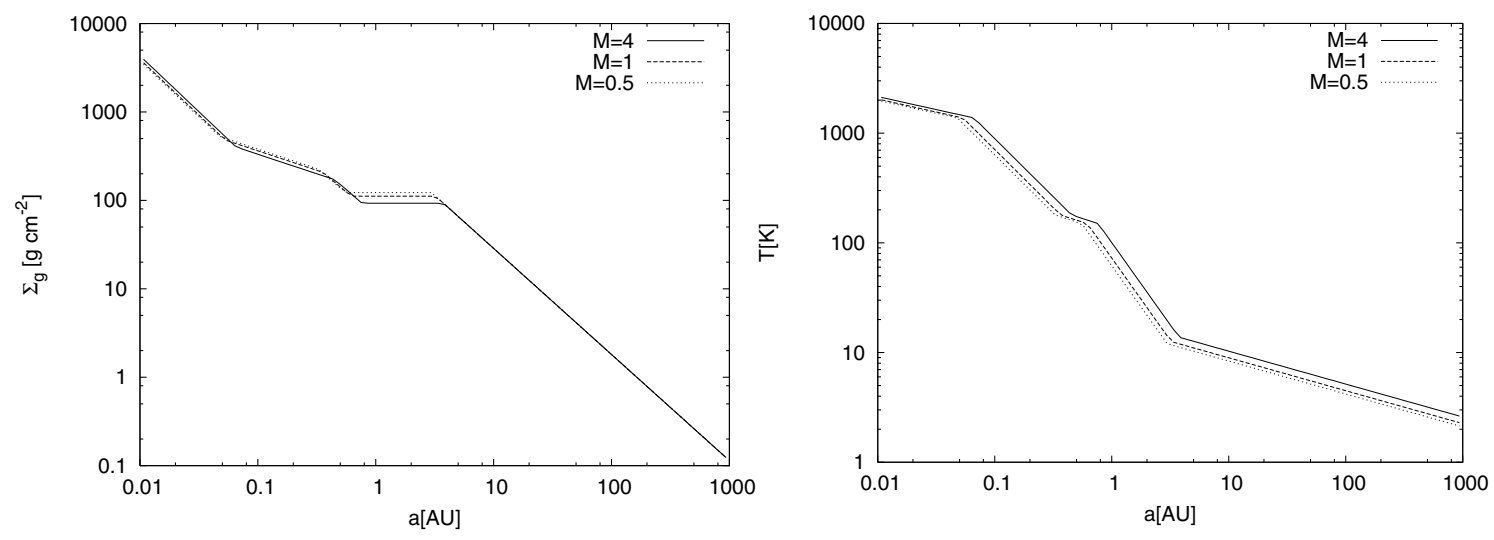

Fig. 1. The initial surface density (left panel) and temperature (right panel) as a function of distance from the star in the protoplanetary disk with an initial mass $M_{0}=0.1 M_{\odot}$ and outer radius $R_{0}=1000 \mathrm{AU}$. Different curves correspond to different values of the mass of the central star in solar masses as described by the labels.

where $\Sigma_{\mathrm{s}, \text { init }}$ is the initial surface density of planetesimals, and $\Delta a$ is the width of the feeding zone. Herein we assume that $\Delta a=$ $8 R_{\mathrm{H}}$ (Lissauer 1993).

To calculate the rate of gas accretion onto the protoplanet, one should solve the equations of mass conservation, hydrostatic equilibrium, energy generation from accretion of planetesimals and quasi-static contraction, and radiative or convective energy transport, as given e.g. in Bodenheimer \& Pollack (1986). Following Ida \& Lin (2004a), we use a simplified approach based on fits to the numerical results. We assume that the accretion of gas starts when the core reaches critical mass of

$M_{\mathrm{c}, \text { crit }}=10\left(\frac{\dot{M}_{\mathrm{c}}}{10^{-6} M_{\oplus} \mathrm{yr}^{-1}}\right)^{0.2-0.3}\left(\frac{\kappa_{\mathrm{env}}}{1 \mathrm{~cm}^{2} \mathrm{~g}^{-1}}\right)^{0.2-0.3}$

(Ikoma et al. 2000), where $\kappa_{\text {env }}$ is the opacity in the envelope of the planet. Its actual magnitude is currently poorly constrained. We assume that $\kappa_{\text {env }}=1 \mathrm{~cm}^{2} \mathrm{~g}^{-1}$. For the value of the powerlaw index in the dependence on $M_{\mathrm{c}}$ (Eq. (10)) we adopt 0.25 . When the mass of the protoplanet is higher than $M_{\mathrm{c}, \text { crit }}$ it contracts on the Kelvin-Helmholtz time scale $\tau_{\mathrm{KH}}$. Bryden et al. (2000) show by fitting the result of Pollack et al. (1996) that

$\tau_{\mathrm{KH}}=10^{b}\left(\frac{M_{\mathrm{p}}}{M_{\oplus}}\right)^{-c+1}\left(\frac{\kappa}{1 \mathrm{~cm}^{2} \mathrm{~g}^{-1}}\right) \mathrm{yr}$

where $b=10$ and $c=-4$. Consequently, for $M_{\mathrm{p}}>M_{\mathrm{c}, \text { crit }}$ we adopt the following equation for the gas accretion rate onto the planet

$\frac{\mathrm{d} M_{\mathrm{env}}}{\mathrm{d} t}=\frac{M_{\mathrm{p}}}{\tau_{\mathrm{KH}}}=A\left(\frac{M_{\mathrm{p}}}{M_{\oplus}}\right)^{-c} M_{\oplus} \mathrm{yr}^{-1} \equiv \tilde{A} M_{\mathrm{p}}^{-c}$,

where $A=10^{-10}$. The free parameters in Eqs. (7)-(12) are the mass of the central star $M_{\star}$, the distance $a$ of protoplanet from the star, and the initial surface density of planetesimals $\Sigma_{\mathrm{s}, \text { init }}$.

\section{Results}

In the CAGCM the process of planet formation is naturally split into two main phases. In the first one, dominated by collisional accumulation of dust grains, a planetesimal swarm is formed in the protoplanetary disk. In the second phase, dominated by gravitational interactions, planetary cores are assembled and subsequently accrete planetesimals and gas from the disk. In the following subsections we investigate how each phase is influenced by the mass of the central star.

\subsection{From dust grains to planetesimals}

To illustrate how the mass of the central star influences the formation of the planetesimal swarm, we follow the evolution of a protoplanetary disk with an initial mass $M_{0}=0.1 M_{\odot}$ and initial outer radius $R_{0}=1000 \mathrm{AU}$ for three values of $M_{\star}(0.5,1$ and $\left.4 M_{\odot}\right)$. Figure 1 shows the initial distributions of $\Sigma_{\mathrm{g}}$ and $T$ in the midplane of the disk. In all three cases the gas is distributed very similarly, with $\Sigma_{\mathrm{g}}$ dropping monotonically from $\sim 4000 \mathrm{~g} \mathrm{~cm}^{-2}$ at $0.01 \mathrm{AU}$ from the star to $0.1 \mathrm{~g} \mathrm{~cm}^{-2}$ at the outer edge of the disk. The changes of slopes in the distribution of $\Sigma_{\mathrm{g}}$ correspond to transitions between different powerlaws describing the opacity of the disk matter in different temperature ranges (Ruden \& Pollack 1991). The distribution of temperature is qualitatively very similar to the distribution of $\Sigma_{\mathrm{g}}$. In the disk around a $1 M_{\odot}$ star $T$ drops from $2500 \mathrm{~K}$ at $0.01 \mathrm{AU}$ to a few Kelvins at the outer edge of the disk. The evaporation temperature assumed for the dust grains in our models $(1350 \mathrm{~K})$ is reached at $0.05 \mathrm{AU}$. Note that at a given radius $T$ increases as the mass of the central star is increased. It is the result of the increasing vertical component stellar gravity, due to which the scale height of the disk is reduced.

Initially, the dust is well mixed with the gas, with the ratio $\Sigma_{\mathrm{s}} / \Sigma_{\mathrm{g}}=6 \times 10^{-3}$ being constant everywhere in the disk. As the disk evolves, surface density and temperature of gas slowly decrease due to accretion and viscous spreading; however, the dust component evolves in quite a different way. The grains grow in size due to mutual collisions and gain inward radial velocities due to the gas drag. If they cross the evaporation radius, they sublimate and are accreted onto the star on the viscous timescale as a vapour. However, if their growth time is shorter than the timescale of inward migration, they manage to reach sizes of a few $\mathrm{km}$ before reaching the evaporation radius. Their radial motions are then stopped and the planetesimal swarm attains its final form. Figure 2 shows the distribution of planetesimals in our model after $10^{6} \mathrm{yr}$ from the beginning of its evolution. In all three cases, the outer radius of the planetesimal swarm is much smaller than the initial outer radius of the disk. The difference is larger for models with smaller $M_{\star}$. This is because small solid bodies evolving in disks around less massive stars gain higher inward velocities and tend to migrate to smaller radius before reaching km-sizes. The maximum velocity of the inward drift can be estimated as

$V_{\mathrm{s}}^{\max } \sim \frac{C_{\mathrm{s}}^{2}}{a \Omega_{\mathrm{K}}}$ 


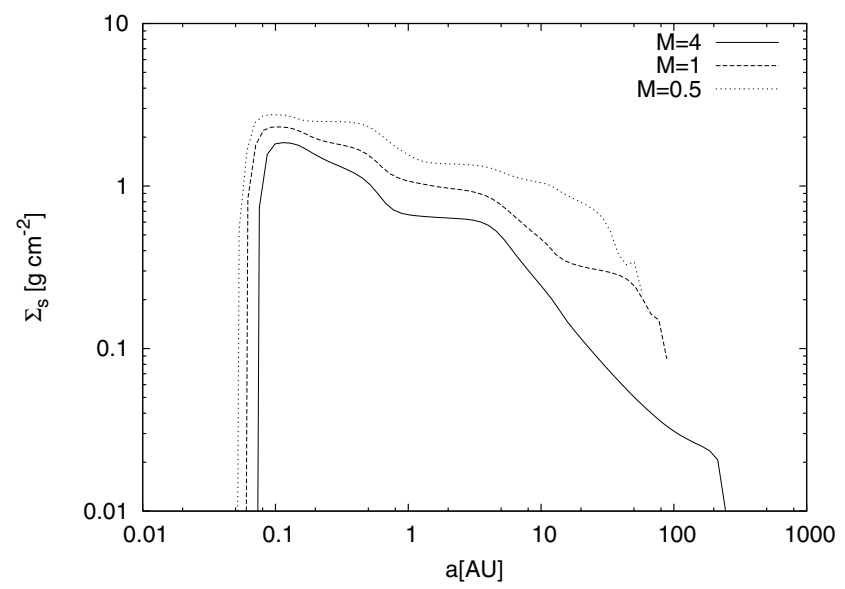

Fig. 2. The surface density of solids as a function distance from the star in the protoplanetary disk with the same parameters as in Fig. 1 after $10^{6} \mathrm{yr}$ from the beginning of its evolution. Different curves correspond to different values of the mass of the central star in solar masses as described by the labels.

(Weidenschilling 1977; Kornet et al. 2002). A change $M_{\star}$ influences it in two opposite manners. On the one hand, $V_{\mathrm{s}}^{\max }$ increases with the mass of the star due to the increase in the disk temperature. On the other hand, it decreases because of the increase in the Keplerian velocity. Of these two competing factors, the second one dominates, and as a result the $V_{\mathrm{s}}^{\max }$ is a decreasing function of $M_{\star}$.

Due to the inward migration of solids and their confinement to much smaller radii, the final surface density of planetesimals is increased locally within a factor of a few in comparison with the initial value of $\Sigma_{\mathrm{s}}$. As this effect is larger in disks around less massive stars, their final planetesimal swarms tend to be more favourable to the formation of giant planets.

\subsection{Minimum surface density}

To quantify the influence of $M_{\star}$ on the effectiveness of giant planet formation from a planetesimal swarm, we introduce the concept of the minimum surface density $\Sigma_{\mathrm{s}, \min }$. We define it as the minimum value of the initial surface density of planetesimals $\Sigma_{\mathrm{s} \text {,init }}$ needed to form a Jupiter-mass $\left(1 M_{\mathrm{J}}\right)$ planet in less than the lifetime of the protoplanetary disk $\tau_{\mathrm{f}}$. For $\tau_{\mathrm{f}}$ we adopt a value of $3 \times 10^{6} \mathrm{yr}$.

First, by solving the set of Eqs. (7), (9), and (12) with different values of $\Sigma_{\mathrm{s} \text {,init }}$, we determine $\Sigma_{\mathrm{s} \text {,min }}$ as a function of distance from the star. The results are shown in Fig. 3 for the same values of $M_{\star}$ as before. Close to the star $(a<10 \mathrm{AU}), \Sigma_{\mathrm{s}, \text { init }}$ is a decreasing function $a$. In this regime, the accreting protoplanetary core rapidly accumulates all planetesimals in its feeding zone and reaches the isolation mass $M_{\text {iso }}$. Afterwards, the accretion of planetesimals is negligible and the planet grows mainly due to the accretion of gas. Upon integrating Eq. (12), we obtain the minimum isolation mass needed to form a $1 M_{\mathrm{J}}$ planet within $\tau_{\mathrm{f}}$ :

$M_{\text {iso }} \geq\left[M_{\mathrm{J}}^{1-c}+(c-1) \tilde{A} \tau_{\mathrm{f}}\right]^{1 /(1-c)}$,

where we assumed that the time needed for the core to reach $M_{\text {iso }}$ is much shorter than $\tau_{\mathrm{f}}$. On the other hand, from the definition of $M_{\text {iso }}$, we see that

$M_{\text {iso }}=\frac{(2 \pi)^{3 / 2}}{\sqrt{h}} a^{3}\left(\frac{\Delta a}{R_{\mathrm{H}}}\right)^{3 / 2} M_{\star}^{-1 / 2} \Sigma_{\mathrm{s}, \text { init }}^{3 / 2}$.

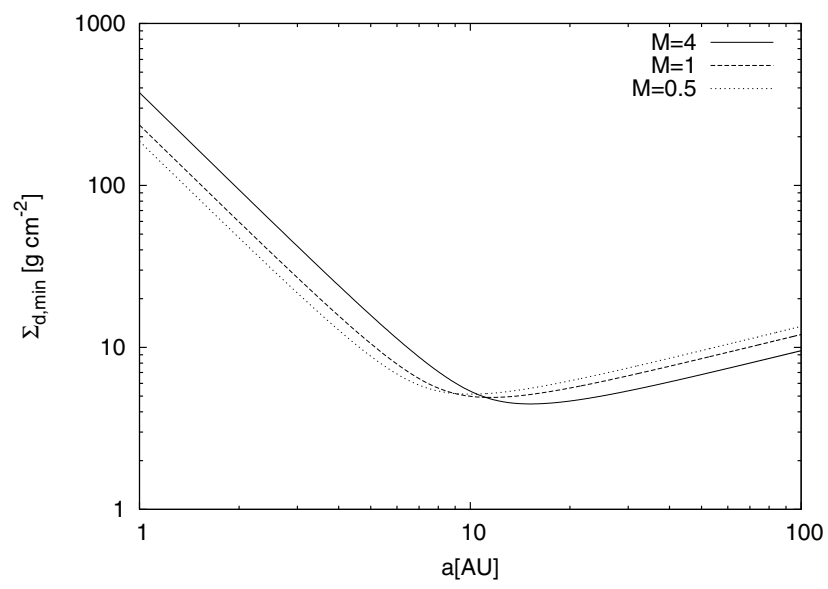

Fig. 3. The minimum surface density of a planetesimal swarm needed to from a $1 M_{\mathrm{J}}$ planet in less than $3 \times 10^{6} \mathrm{yr}$ as a function of distance from the central star. Different curves are obtained for different masses of the central star, as labeled in units of solar masses in the upper right corner.

Combining these two equations and neglecting the factor $M_{\mathrm{J}}^{1-b}$, we obtain an analytical formula for $\Sigma_{\mathrm{s}, \mathrm{min}}$

$\Sigma_{\mathrm{s}, \min }=\frac{h^{1 / 3}}{2 \pi} a^{-2}\left(\frac{\Delta a}{R_{\mathrm{H}}}\right)^{-1} M_{\star}^{1 / 3}\left[(c-1) \tilde{A} \tau_{\mathrm{f}}\right]^{2 / 3(1-c)}$,

which for $a<10$ AU agrees very well with the exact values of $\Sigma_{\mathrm{s}, \min }$ on the descending branch of the curve $\Sigma_{\mathrm{s}, \min }(a)$ (see Fig. 4). Note that in this regime the quantity $\Sigma_{\mathrm{s}, \min }$ is an increasing function of the stellar mass. This behaviour is due to the fact that, while we increase $M_{\star}$, the Hill sphere shrinks, and as a result the isolation mass decreases (provided, of course, that $\Sigma_{\mathrm{s}, \text { init }}$ stays constant).

For sufficiently large radii ( $a>10 \mathrm{AU}), \Sigma_{\mathrm{s}, \min }(a)$ changes its slope and becomes an increasing function of $a$. In this regime, the time scale of accretion of the planetesimals onto the core is larger than the lifetime of the disk and becomes the main factor to determine $\Sigma_{\mathrm{s} \text {, min }}$. Consequently, the surface density of planetesimals in the feeding zone never drops much below its initial value. To describe the formation of a planet analytically under these conditions, we divide the whole process into two phases. During the first phase the planet exclusively due to the accretion of planetesimals grows. We assume that the surface density of planetesimal swarm $\Sigma_{\mathrm{s}}$ does not change in time, because the core only accumulates a negligible fraction of solids present in the feeding zone. From Eq. (7) we obtain

$\frac{\mathrm{d} M_{\mathrm{p}}}{\mathrm{d} t}=A_{\mathrm{c}} M_{\mathrm{p}}^{2 / 3}$

where

$A_{\mathrm{c}}=\left(\frac{3 \pi^{2}}{4}\right)^{1 / 3} \sqrt{G} C_{1} C_{\mathrm{cap}} \frac{1}{\left(h \rho_{\mathrm{c}}\right)^{1 / 3}} \frac{M_{\star}^{1 / 6}}{\sqrt{a}} \Sigma_{\mathrm{s}, \text { init }}$

is a constant factor (we neglect the changes of $C_{\text {cap }}$ in time, and $\rho_{\mathrm{c}}$ in the above expression denotes the density of the core). During the second phase the planet grows exclusively due to the accretion of gas at a rate described by Eq. (12). This phase begins when the planet reaches the mass $M_{\mathrm{p} \text {,int }}$ for which the accretion rate of gas is equal to the accretion rate of planetesimals. From Eqs. (12) and (17), we get

$M_{\mathrm{p}, \text { int }}=\left(\frac{A_{\mathrm{c}}}{\tilde{A}}\right)^{3 /(3 c-2)}$. 


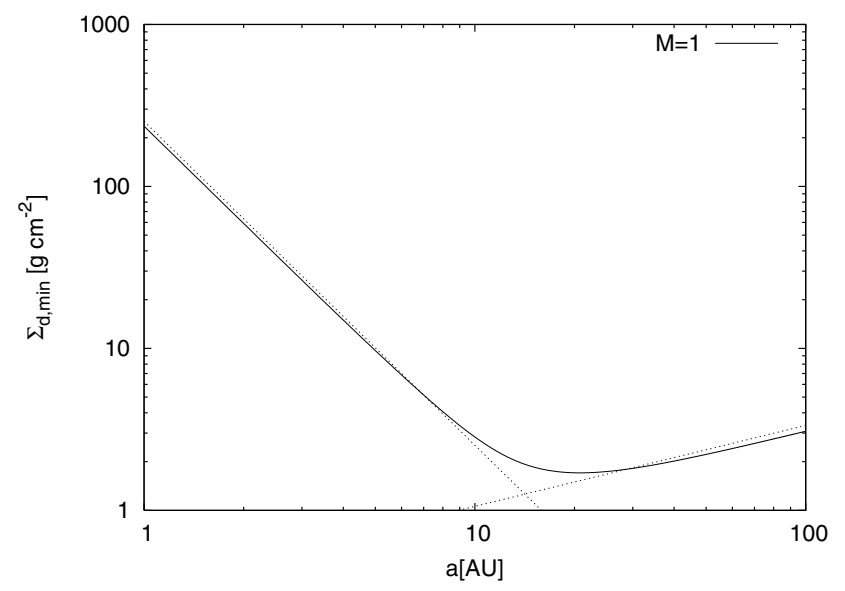

Fig. 4. The minimum surface density of the planetesimal swarm needed to form a $1 M_{\mathrm{J}}$ planet in less than $3 \times 10^{6} \mathrm{yr}$ as a function of distance from the central star, calculated with a constant value of $C_{\text {cap }}=5$. The solid line represents the results of exact values obtained numerically, while dotted lines represent analytical approximations given by Eqs. (16) and (22). The mass of the central star is $1 M_{\odot}$.

Integrating Eqs. (17) and (12) over time yields the lengths of both phases $\Delta t_{1}$ and $\Delta t_{2}$ :

$\Delta t_{1}=\frac{3 M_{\mathrm{p}, \text { int }}^{1 / 3}}{A_{\mathrm{c}}}$,

and

$\Delta t_{2}=\frac{M_{\mathrm{p}, \text { int }}^{1-b}}{(b-1) \tilde{A}}$.

Combining Eqs. (18)-(21) with the condition that $\Delta t_{1}+\Delta t_{2} \leq \tau_{\mathrm{f}}$, we get the equation for $\Sigma_{\mathrm{s}, \min }$ in that regime:

$\Sigma_{\mathrm{s}, \min }=\left(\frac{4}{3 \pi^{2}}\right)^{1 / 3} \frac{\left.\left(h \rho_{\mathrm{c}}\right]\right)^{1 / 3}}{\sqrt{G} C_{1} C_{\mathrm{cap}}} \tilde{A}^{\frac{1}{3(1-c)}}\left[\frac{c-1}{3 c-2} \tau_{\mathrm{f}}\right]^{\frac{3 c-2}{3(1-c)}} \frac{\sqrt{a}}{M_{\star}^{1 / 6}}$.

With $C_{\text {cap }}=1.2$, the above formula satisfactorily reproduces the rising branch of curve $\Sigma_{\mathrm{s}, \min }(a)$ (see Fig. 4). Note that the "bestfit" value of $C_{\text {cap }}$ is consistent with the maximum and minimum values of this parameter used in our numerical calculations (1 and 5, respectively). At large distances from the star, $\Sigma_{\mathrm{s}, \min }$ is a decreasing function of $M_{\star}$, because increasing the mass of the star causes the accretion rate of planetesimals to decrease (see Eq. (17)).

As we see, the minimum surface density of the planetesimal swarm required for the formation of a gas giant planet in a time shorter than the lifetime of protoplanetary disk is a complicated function of $M_{\star}$. In a given planetesimal swarm such a planet forms more easily around less massive star if its orbital radius is smaller than $\sim 10 \mathrm{AU}$, and around a more massive star if its orbital radius is larger than $\sim 10 \mathrm{AU}$.

\subsection{Grid of models}

The results of the last two subsections allow us to investigate the influence of $M_{\star}$ on the whole process of giant planet formation for a broad set of models of protoplanetary disks. We calculate the grid of models similar to the one described in Sect. 3.1, but with different values of the initial disk mass $M_{0}$ and outer radius $R_{0}$. To cover the range of masses and sizes of disks observed

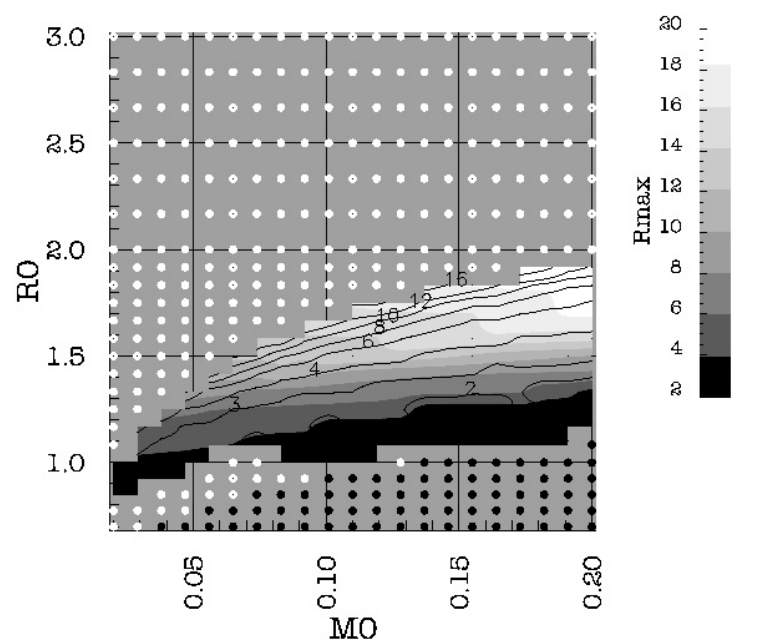

Fig. 5. The plane of initial parameters of protoplanetary disk models $\left[M_{0}, \log R_{0}\right]$. Minimum and maximum distance from a $1 M_{\odot}$ star at which the formation of a $1 M_{\mathrm{J}}$ planet is possible within $3 \times 10^{6} \mathrm{yr}$ is indicated by contours and a grey scale, respectively. White circles indicate disk models in which the surface density of the planetesimal swarm is everywhere lower than the critical value for planet formation. Black circles indicate disks in which all solids are accreted onto the star.

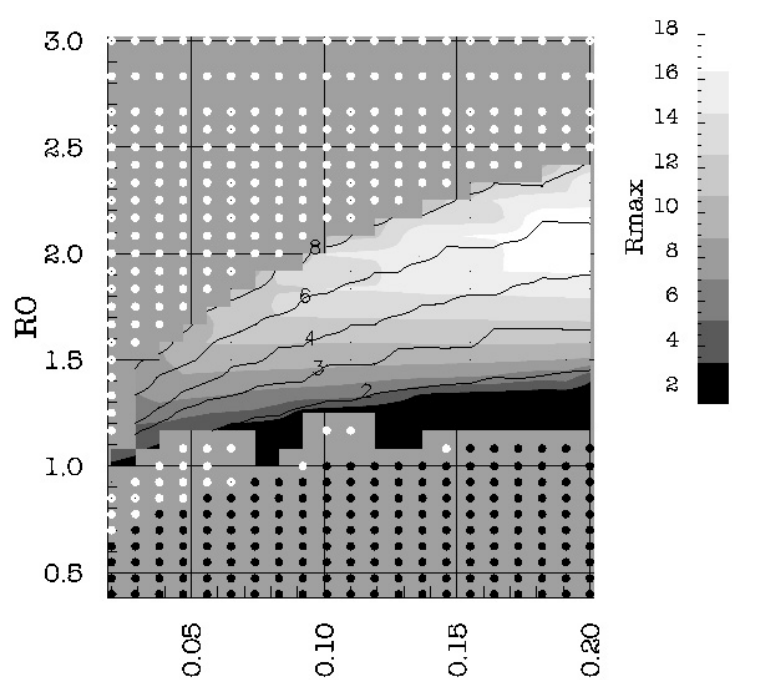

Fig. 6. Same as Fig. 5 but for the central star with a mass of $0.5 M_{\odot}$.

in nature, we choose $M_{0}$ in the range of 0.02 to $0.2 M_{\odot}$. The range of $R_{0}$ is adjusted for every metallicity so that all models in which formation of giant planets is possible could be accounted for. For the viscosity coefficient $\alpha$ we chose a value of 0.001 (Papaloizou \& Nelson 2003). We follow each model until all solids are in the form of planetesimals or are accreted onto the star. Then, we evaluate every model with planetesimals to determine whether the surface density of planetesimals exceeds the minimum surface density $\Sigma_{\mathrm{s}, \min }$ anywhere in the disk. Models with this property are labeled as planet bearing. For each such model we determine the minimum and maximum distances from the star at which the surface density of the planetesimal swarm is larger than $\Sigma_{\mathrm{s}, \min }$. The results obtained for different values of the stellar mass are shown in Figs. 5-7.

The area of the region occupied on the $\left[M_{0}, R_{0}\right]$ plane by the planet bearing disks is clearly anticorrelated with the mass of the central star. As we have shown in Sect. 3.1, solid grains gain higher inward velocities in disks around less massive stars, and the resulting planetesimal swarms have higher surface densities. 


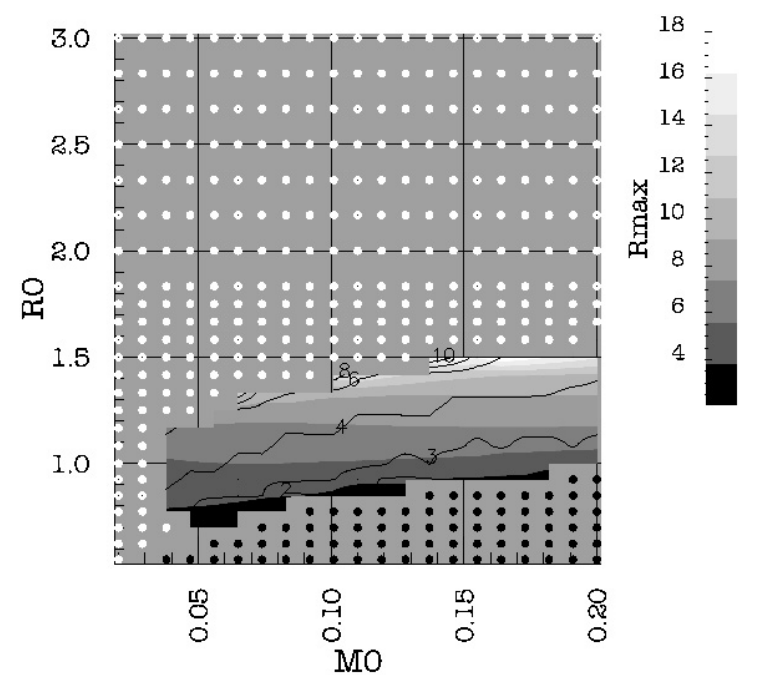

Fig. 7. Same as Fig. 5 but for the central star with a mass of $4 M_{\odot}$.

We see that reducing the mass of the central star increases the maximum $R_{0}$ for which planet formation is possible in disks with the same initial mass $M_{0}$.

We can also see that in a given disk the inner edge of the planet-bearing region moves inward as we decrease the mass of the star (for $M_{\star}=0.5 M_{\odot}$ its radius is on the average equal to $\sim 0.55$ that for $M_{\star}=1 M_{\odot}$ ). This effect is mainly caused by the larger surface density of planetesimal swarms produced by disks around less massive stars. Differences in $\Sigma_{\mathrm{s} \text {,min }}$, while appreciable, are much less important (when $M_{\star}$ is reduced by a factor of $2, \Sigma_{\mathrm{s}, \min }$ drops by $20 \%$ only; see Eq. (16)). The outer edge of the planet-bearing region also moves closer to the star (for $M_{\star}=0.5 M_{\odot}$ its radius is on the average equal to $\sim 0.75$ that for $\left.M_{\star}=1 M_{\odot}\right)$. This is because in most cases it coincides with the outer edge of the planetesimal swarm, which is more compact around less massive stars. The difference in the minimum surface density $\Sigma_{\mathrm{s} \text {, min }}$ also tends to decrease the outer radius of the planet-bearing region, but it is again a second-order factor.

Generally, our model predicts that giant planets tend to form at tighter orbits around less massive stars, and wider orbits around more massive stars. However, at least in some cases their locations may be influenced by the effects of migration. We return to this point in Sect. 4.

\subsection{Metallicity relation}

One of the main results of extrasolar planet searches is the discovery that planet-bearing stars tend to have higher metallicities than field stars (Santos et al. 2000; Fischer \& Valenti 2003). That correlation can be easily explained within CAGCM. In this scenario, the formation time of giant planets decreases with increasing surface density of the planetesimal swarm (see Eqs. (16) and (22)), which in turn is an increasing function of the original metal content of the protoplanetary disk. Consequently, the giant planets form more easily in disks with higher metallicities. Kornet et al. (2005) calculated the rates of giant-planet occurrence in disks with different metallicities around stars with mass $M_{\star}=1 M_{\odot}$. Their approach to the evolution of solids and to the formation of giant planets was the same as the one used in this paper. They were able to reproduce the observational correlation for disk models with viscosity parameter $\alpha=10^{-3}-10^{-2}$. Herein we extend their calculations onto disks around stars with various masses.
The change in the disk metallicity influences the processes leading to the formation of planets in two ways. First, it changes the structure of the gaseous disk by changing the opacity in the disk. In our models we scaled the opacity by a constant factor $Z$ equal to the metallicity of the disk expressed in solar units. This approach is justified by the fact that the opacity in protoplanetary disks is mainly due to dust grains and molecules. Second, the primordial metallicity of the disk determines the initial ratio of dust-to-gas surface densities. In our models this ratio is initially independent of the distance from the star and is equal to

$\Sigma_{\mathrm{s}} / \Sigma_{\mathrm{g}}=6 \times 10^{-3} Z$

We compute grids of models similar to those described in Sect. 3.3 for eight different values of $Z$ distributed between 0.2 and 3. Additionally, we check the gravitational stability of the corresponding gaseous disk for every value of $\left[M_{0}, R_{0}\right]$. In some cases the value of the Toomre parameter

$Q=\frac{C_{\mathrm{S}} \Omega_{\mathrm{K}}}{\pi G \Sigma}$,

where $\Sigma=\Sigma_{\mathrm{g}}+\Sigma_{\mathrm{s}}$, drops below 1 in the outer region of the disk, which means that they are unstable with respect to axisymmetric modes. We assume that such region fragments and giant planets are formed there on a very short time scale, consuming and/or dispersing the unstable part of the disk. In such cases we use modified initial values of $M_{0}$ and $R_{0}$, which correspond to the mass and outer radius of the stable part of the original disk. In principle, some "special treatment" should also be applied to disks with $1 \leq Q \lesssim 1.3$, which develop spiral arms and for a while evolve due to gravitational rather than viscous angular momentum transfer (see e.g. Laughlin \& Rozyczka 1996). However, since the efficiency of gravitational transport quickly decreases with the ratio $M_{0} / M_{\star}$ and it might become significant in our study only for the most massive disks around $0.5 M_{\odot}$ stars, we decided to neglect this effect altogether.

Following the procedure described by Kornet et al. (2005), for every $Z$ we calculate the area $A_{\mathrm{p}, 5}$ of the region occupied on the $\left[M_{0}, \log R_{0}\right]$ plane by disks that form planets at distances smaller than $5 \mathrm{AU}$ from the central star. The last restriction comes from the fact that currently we know only one extrasolar planet on a larger orbit ${ }^{1}-55 \mathrm{Cnc} d$ (Marcy et al. 2002). A measure of the rate of planet occurrence can be defined as

$P_{\mathrm{p}}=\frac{A_{\mathrm{p}, 5}}{C}$,

where the normalization factor $C$ is chosen in such a way as to reproduce the observed value of $P_{\mathrm{p}}$ for $Z=3$ and $M_{\star}=1 M_{\odot}$.

The results are presented in Fig. 8. It shows the rate of planet occurrence as a function of disk metallicity for three values of $M_{\star}$. As expected, $P_{\mathrm{p}}$ is an increasing function of $Z$. The minimum value of $Z$ below which no giant planets are formed at orbits smaller than $5 \mathrm{AU}$ decreases with the mass of the central from $Z_{\text {min }} \approx 0.6$ for $M_{\star}=0.5 M_{\odot}$, to $Z_{\min } \approx 0.2$ for $M_{\star}=4 M_{\odot}$.

We see that for $Z$ smaller than $\sim 0.2, P_{\mathrm{p}}$ is an increasing function of $M_{\star}$. This is because most disks in which planets formation would be possible around less massive stars have outer parts that are gravitationally unstable, and the amount of solids present in their stable parts is too low to enable subsequent formation of giant planets according to CAGCM. However, as $Z$ increases, smaller and smaller disks become planet-bearing for every $M_{0}$,

\footnotetext{
1 see The Extrasolar Planets Encyclopedia at http://www . obspm. fr/encycl/encycl.html
} 


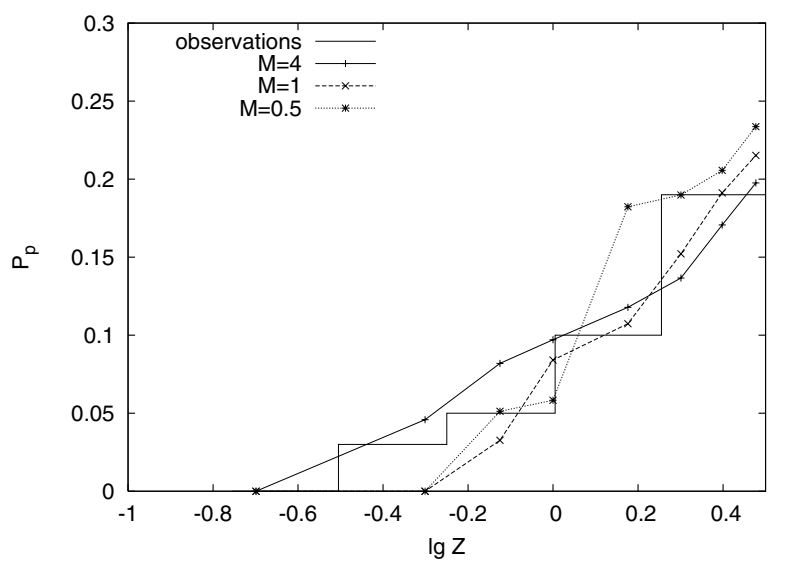

Fig. 8. The rate of planet occurrence as a function of the primordial metallicity of protoplanetary disks. Different lines are obtained for models with different masses of the central star, as labeled in solar units in the upper left corner. The histogram shows the observational data compiled by Fischer \& Valenti (2003).

and the percentage of gravitationally unstable disks in which formation of giant planets is not possible decreases. Consequently, the factors promoting the formation of a giant planet around less massive stars as described in previous sections become important, and $P_{\mathrm{p}}$ changes into a decreasing function of $M_{\star}$.

\section{Conclusions}

Based on a simple approach to the evolution of solids in protoplanetary disks, we investigated the influence of the mass of the central star on the formation of giant planets. We showed that due to the more efficient redistribution of solids the planetesimal swarms around less massive stars tend to have higher surface densities. Next, we derived the minimum surface density of the planetesimal swarm needed to enable formation of a giant planet within the lifetime of the protoplanetary disk, and we found that at distances from the star smaller than $\sim 10 \mathrm{AU}$ it increases with the stellar mass. Farther away from the star the minimum density becomes anticorrelated with the mass of the star. However this effect is offset by the anticorrelation mentioned already between the mass of the star and the surface density of the planetesimals.

These two effects determine the set of initial parameters characterising protoplanetary disks that are capable of giant planet formation within the core accretion - gas capture scenario. We showed that this set is larger for less massive stars. This means that the percentage of stars with massive planets should increase with decreasing stellar mass (at least in the range 0.5-4 $\left.M_{\odot}\right)$. However, as discussed below, in the currently accessible range of orbital radii $(<5 \mathrm{AU})$, the situation is not all that clear.

Based on the sets obtained for different metallicities, we determined the occurrence rate of planets with orbits smaller than $5 \mathrm{AU}$ as a function of the mass and metallicity of the star. We took into account the fact that the outer region of the disk is gravitationally unstable in some models. Such regions are located farther than $5 \mathrm{AU}$ from the central star, and planets formed there by disk fragmentation are not included in our occurrence rate. However, their presence reduces the amount of solid material available for the formation of planetesimals. For less massive stars this effect is so strong that it overcomes factors promoting planet formation, so that for metal-poor disks the rate of planet occurrence decreases with the mass of the central star. As a

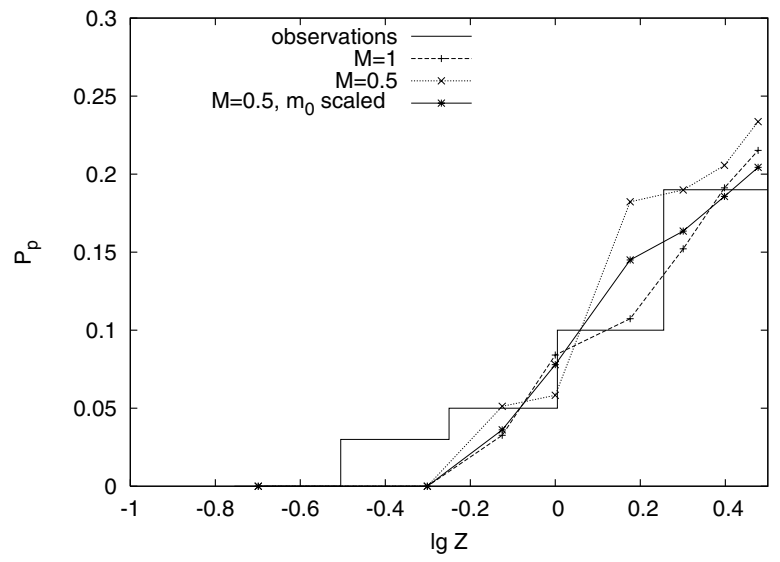

Fig. 9. The rate of planet occurrence as a function of the primordial metallicity of protoplanetary disks. Disks around stars with masses of $1 M_{\odot}$ are represented by a solid line. Disks around stars with masses of $0.5 M_{\odot}$ are represented by dotted and dashed lines. In the first case the range of initial masses of the disks is the same as in the case of solar type stars, while in the second it was scaled according to the mass of the central star.

result, the minimum metallicity at which giant planets can form at orbits smaller than $5 \mathrm{AU}$ decreases from $\sim 0.6$ for stars with masses of $0.5 M_{\odot}$ to $\sim 0.2$ for $4 M_{\odot}$.

In the metal-rich regime the percentage of entirely stable disks in which formation of giant planets is possible is larger, and stable regions of partly unstable disks contain enough solids to produce planetesimal swarms capable of giant planet formation. Consequently, both factors promoting planet formation around less massive stars are in play, and a clear anticorrelation between the stellar mass and planet occurrence rate is observed. At the same time, our model does not account for the presence of giant planets around metal-poor stars. This may be due to the fact that we do not include planets that have formed beyond $5 \mathrm{AU}$ and later migrated inward. Such an assumption is valid as long as the number of these planets is small compared to the number of planets that have formed within 5 AU. However, as we move to lower metallicities, the percentage of giant planets with silicate cores decreases (the silicates simply become too scarce), while the percentage of planets forming from ice grains increases. Thus, in metal-poor systems the number of planets with ice cores that migrated from large orbits can become a large fraction of planets at orbits smaller than $5 \mathrm{AU}$.

Obviously, our description of the evolution of solids is very simplified. The basic underlying assumptions like the singlesize distribution of solid grains or the neglect of planet migration already have been discussed by Kornet at al. (2004, 2005). The main additional assumption introduced in the present paper is the independence of the initial parameters of protoplanetary disks on the mass of the central star. While admittedly ad hoc, it seems to be better than the one adopted by Laughlin et al. (2004), who scaled their initial surface density of planetesimals linearly with the mass of the star. They did not take into account the antecedent evolution of solids leading to the formation of planetesimal swarms, and concluded that the formation of giant planets around low-mass stars is difficult. Recent observations suggest that masses of protoplanetary disks do not strongly depend on masses of the central stars (Guilloteau 2005). Nevertheless, to investigate the influence of our assumption, we performed additional set of calculations with a mass of the central star of $0.5 M_{\odot}$ and with the initial masses of disks scaled by factor of 0.5 . The results are shown in Fig. 9. In this case the probability of finding 
a planet does not seem to depend strongly on the mass of the central star, which is true for the whole range of metallicities we have considered. Still, our models show that the evolution of solids leading to the formation of planetesimal swarms is a vital factor facilitating the formation of giant planets, whose role should be particularly clear for low-mass stars.

Our models of gaseous disks do not reproduce recent observations by (Muzerolle et al. 2005), which show that the accretion rate in protoplanetary disks increases with the mass of the central star. However, in the mass range considered here this dependence is very weak, and for a given value of stellar mass the spread in accretion rates reaches two orders of magnitude. In our opinion these data do not invalidate our basic assumption that initial disk parameters do not depend on the mass of the star. We also assumed that heating by stellar radiation is negligible, whereas at least in some cases it can be a dominant source of energy in the outer regions of the disk (more efficient than the turbulent dissipation). As such, it may substantially change the structure of the disk and the radial velocities of solids. Currently we are working on models that will take these effects into account.

Acknowledgements. This project was supported by the German Research Foundation (DFG) through the Emmy Noether grant WO 857/2-1 and the European Community's Human Potential Programme through the contract HPRN-CT-2002-00308, PLANETS. K.K. and M.R. acknowledge support from the grant No. 1 P03D 02626 from the Polish Ministry of Science.

\section{References}

Bodenheimer, P., Hubickyj, O., \& Lissauer, J. J. 2000, Icarus, 143, 2 Bodenheimer, P., \& Pollack, J. B. 1986, Icarus, 67, 391

Bonfils, X., Delfosse, X., Udry, S., et al. 2004, in ASP Conf. Ser., 101

Bryden, G., Lin, D. N. C., \& Ida, S. 2000, ApJ, 544, 481

Butler, R. P., Vogt, S. S., Marcy, G. W., et al. 2004, ApJ, 617, 580
Endl, M., Cochran, W. D., Tull, R. G., \& MacQueen, P. J. 2003, AJ, 126, 3099 Fischer, D., \& Valenti, J. A. 2003, in Scientific Frontiers in Research on Extrasolar Planets, ed. D. Deming, \& S. Seager (San Francisco: ASP), ASP Conf. Ser., 294, 117

Frank, J., King, A., \& Raine, D. 1992, Accretion Power in Astrophysics. (Cambridge University Press)

Guilloteau, S. 2005, private communication

Hubickyj, O. 2001, private communication

Ida, S., \& Lin, D. N. C. 2004a, ApJ, 604, 388

Ida, S., \& Lin, D. N. C. 2004b, ApJ, 616, 567

Ikoma, M., Nakazawa, K., \& Emori, H. 2000, ApJ, 537, 1013

Kornet, K., Bodenheimer, P., Różyczka, M., \& Stepinski, T. F. 2005, A\&A, 430, 1133

Kornet, K., Stepinski, T. F., \& Różyczka, M. 2001, A\&A, 378, 180

Kornet, K., Bodenheimer, P., \& Różyczka, M. 2002, A\&A, 396, 977

Kornet, K., Różyczka, M., \& Stepinski, T. F. 2004, A\&A, 417, 151

Laughlin, G., Bodenheimer, P., \& Adams, F. C. 2004, ApJ, 612, L73

Laughlin, G., \& Rozyczka, M. 1996, ApJ, 456, 279

Lissauer, J. J. 1993, ARA\&A, 31, 129

Marcy, G. W., Butler, R. P., Vogt, S. S., Fischer, D., \& Lissauer, J. J. 1998, ApJ, 505, L147

Marcy, G. W., Butler, R. P., Fischer, D., et al. 2001, ApJ, 556, 296

Marcy, G. W., Butler, R. P., Fischer, D. A., et al. 2002, ApJ, 581, 1375

Marcy, G. W., Butler, R. P., Vogt, S. S., et al. 2005, ApJ, 619, 570

Mayor, M., Udry, S., Naef, D., et al. 2004, A\&A, 415, 391

Muzerolle, J., Luhman, K. L., Briceño, C., Hartmann, L., \& Calvet, N. 2005, ApJ, 625, 906

Papaloizou, J. C. B., \& Nelson, R. P. 2003, MNRAS, 339, 983

Papaloizou, J. C. B., \& Terquem, C. 1999, ApJ, 521, 823

Podolak, M., Pollack, J. B., \& Reynolds, R. T. 1988, Icarus, 73, 163

Pollack, J. B., Hubickyj, O., Bodenheimer, P., et al. 1996, Icarus, 124, 62

Ruden, S. P., \& Pollack, J. B. 1991, ApJ, 375, 740

Santos, N. C., Israelian, G., \& Mayor, M. 2000, A\&A, 363, 228

Stepinski, T. F. 1998, Icarus, 132, 100

Stepinski, T. F., \& Valageas, P. 1997, A\&A, 319, 1007

Weidenschilling, S. J. 1977, MNRAS, 180, 57

Weidenschilling, S. J. 2003, in Lunar and Planetary Science Conference \#34, abstract \#1707

Weidenschilling, S. J., \& Cuzzi, J. N. 1993, in Protostars and Planets III, ed. E. Levy, \& J. Lunine (Tuscon: Univ. of Arizona Press), 1031 